\title{
Viable Constraint on Scalar Field in Scalar-Tensor Theory
}

\author{
Chao-Qiang Geng, $1,2,3,4, *$ Hao-Jui Kuan, $3,+$ and Ling-Wei Luo ${ }^{5,4}$ \\ ${ }^{1}$ School of Fundamental Physics and Mathematical Sciences \\ Hangzhou Institute for Advanced Study, UCAS, Hangzhou 310024, China \\ ${ }^{2}$ International Centre for Theoretical Physics Asia-Pacific, Beijing/Hangzhou, China \\ ${ }^{3}$ Department of Physics, National Tsing Hua University, Hsinchu 300, Taiwan \\ ${ }^{4}$ Physics Division, National Center for Theoretical Sciences, Hsinchu 300, Taiwan \\ ${ }^{5}$ Institute of Physics, Academia Sinica, Taipei 11529, Taiwan
}

(Dated: April 8, 2020)

\begin{abstract}
The scalar-tensor theory can be formulated in both Jordan and Einstein frames, which are conformally related together with a redefinition of the scalar field. As the solution to the equation of the scalar field in the Jordan frame does not have the one-to-one correspondence with that in the Einstein frame, we give a criterion along with some specific models to check if the scalar field in the Einstein frame is viable or not by confirming whether this field is reversible back to the Jordan frame. We further show that the criterion in the first parameterized post-Newtonian approximation can be determined by the parameters of the osculating approximation of the coupling function in the Einstein frame and can be treated as a viable constraint on any numerical study in the scalar-tensor scenario. We also demonstrate that the Brans-Dicke theory with an infinite constant parameter $\omega_{\mathrm{BD}}$ is a counterexample of the equivalence between two conformal frames due to the violation of the viable constraint.
\end{abstract}

\footnotetext{
* Electronic address: geng@phys.nthu.edu.tw

$\dagger$ Electronic address: guanhauwzray@gmail.com

$\ddagger$ Electronic address: lwluo@gate.sinica.edu.tw
} 


\section{INTRODUCTION}

General Relativity (GR) is a great theory as its predictions agree well with experiments. However, some strong-field phenomena may deviate from those of GR. Jordan proposed a scalar field which couples to the spacetime curvature to fulfill the hypothesis about the variation of the constant of gravitation [1, 2]. Bergmann [3] and Fierz [4] used the KaluzaKlein theory [5] to get an effective gravitational constant. Among various alternative gravity theories, the scalar-tensor (ST) one is the most natural extension to GR since it can be obtained by reducing a higher dimensional theory into a four dimensional one together with a scalar field.

The most famous formulation of the ST theory was done by Brans and Dicke (BD) [6, 7], in which GR is explicitly modified by introducing a scalar field variably to determine the universal Newtonian coupling. Subsequently, the BD theory was generalized by Bergmann [8] and Wagoner [9] to include a scalar potential, which has been widely used to realize inflation

and the late-time cosmic acceleration of the universe. Damour and Esposito-Farse [10] further extended it with couplings between gravity and multi-scalar fields, in which, besides the tensor gravitational field, one or more scalar fields are added in the gravitational sector with non-minimal couplings.

There are two formalisms of the ST theory. One is given in the so called Jordan frame, where the scalar field $\phi$ couples non-minimally to the Ricci scalar $R$ but not directly to matter with the kinetic term for the scalar field involving an arbitrary function $\omega(\phi)$. The other is in the Einstein frame, where the canonical scalar field $\varphi$ is introduced to minimally couple to the Ricci scalar $R^{\star}$. The later one makes the field equations mathematically less complicated due to the separation of the second-order derivatives of the gravitational variables $g_{\mu \nu}^{\star}$ and $\varphi$, but the matter couplings depend on the scalar field. In order to have a frame transformation, we need to assume that each scalar field is a functions of the other. In other words, we should have $\phi(\varphi)$ to rephrase the system from the Jordan frame into the Einstein one, and vice versa. The connection between these two frames is through a Weyl (conformal) transformation by taking the Jordan frame metric $g_{\mu \nu}$ into the Einstein frame one $g_{\mu \nu}^{\star}$, together with a redefinition of the original scalar field $\phi$ into $\varphi$ to have its kinetic term being a canonical form.

The Einstein frame is convenient to perform the numerical simulations and transparent 
to discuss the cosmological applications. However, the issues on the physical interpretation and equivalence of these two frames have been debated for a long time (e.g. [11, 12]). On the other hand, there has been much less attention to the redefinition of the scalar field. In spite of many efforts to show that these two conformal frames are equivalent, in general there are mainly two problems from the redefinition of the scalar field, which are shown as follows:

(i) The existence of the Einstein frame is determined by whether the scalar field $\phi$ can be described in terms of the new field $\varphi$. Furthermore, we need to transform the system back to the Jordan frame. These operations are based on the requirement of the inequalities, given by

$$
\begin{aligned}
& \frac{d \phi}{d \varphi} \neq 0 \quad \text { (Jordam to Einstein frame) } \\
& \frac{d \varphi}{d \phi} \neq 0 \quad \text { (Einstein to Jordam frame) }
\end{aligned}
$$

which are related to each other, Note that the non-zero values of the derivatives should also be finite. We call (1) as the derivative constraints. In practice, one often study the ST theories in the Einstein frame even though most of them do not perceive the inverse problem of the solution in the Jordan frame. In other words, they do not consider the possibility that their results may not be able to be transformed back into the Jordan frame. Since the Jordan frame is assumed to be the physical frame, those fields in this case do not bear any physical meanings. It is one of the purposes of the present work to give an essential criterion to see if a solution in the Einstein frame can uniquely correspond to a Jordan one. It will be shown that (1) leads to the required condition.

(ii) The coefficient of the kinetic term of $\varphi$ determines the value of $d \varphi / d \phi$ through the Weyl transformation. However, the sign of the differential relation $d \varphi / d \phi$ can be either "+" or "-", which has been mentioned in [13] but without further discussions therein, while many just consider the positive sign as in [7, 14, 15]. Therefore, starting from a given ST model in the Einstein frame, it usually corresponds to two different models in the Jordan (physical) frame. We will give an example on this issue. In addition, the condition that the relation $d \varphi / d \phi$ is regular indicates that there is an irrelevant value of $\varphi$ by which the solution space of the scalar fields in the Einstein frame is divided into two branches [16]. Conversely, the value of $\varphi$ can restrict the choice of the sign of the relation $d \varphi / d \phi$ in some cases. As a result, it arises the uniqueness problem of a model in the Jordan frame. 
In this study, we explore the ST theory in the Jordan and Einstein frames by examining the relations between them. In particular, we would like to find out the possible constraints on the theory by requiring the one-to-one correspondence for the physical quantities after the conformal transformation between the two frames.

The paper is organized as follows. In Sec. II, we first write down the ST action. We then display the transformation between the Jordan and Einstein frames and demonstrate that there is a constraint on the scalar transformation. In Sec. III, we take two specific models as examples to illustrate how the constraint manifest itself as a criterion to check if the solution in the Einstein frame is viable or not. We present our conclusions in Sec. IV.

\section{SCALAR-TENSOR THEORIES}

\section{A. Conformal Frame Freedom}

a. 1

A general action of the ST theory with a dimensionless single scalar field can be written

$$
S=\int d^{4} x \frac{\sqrt{-g}}{16 \pi G}\left(F(\phi) R-\mathcal{B}(\phi) g^{\mu \nu} \partial_{\mu} \phi \partial_{\nu} \phi-U(\phi)\right)+S_{m}\left[\psi_{m}, e^{2 \gamma(\phi)} g_{\mu \nu}\right],
$$

where $g$ and $R$ associated with $g_{\mu \nu}$ respectively stand for the determinant and Riemann scalar curvature, $F(\phi), \mathcal{B}(\phi), U(\phi)$ and $\gamma(\phi)$ represent functions of $\phi$ and $\psi_{m}$ denotes the non-gravitational fields. We note that $F(\phi)>0$ in the Jordan frame. The corresponding equations of motion for $g_{\mu \nu}$ and $\phi$ are

$$
R_{\mu \nu}=\frac{1}{F}\left[8 \pi G\left(T_{\mu \nu}-\frac{1}{2} g_{\mu \nu} T\right)+\frac{1}{2} g_{\mu \nu} U+\mathcal{B} \partial_{\mu} \phi \partial_{\nu} \phi\right],
$$

and

$$
\square \phi=\frac{1}{2 \mathcal{B}}\left[-F_{\phi} R+U_{\phi}-\mathcal{B}_{\phi} g^{\mu \nu} \partial_{\mu} \phi \partial_{\nu} \phi-16 \pi G \alpha T\right],
$$

respectively, where the subscript $\phi$ denotes the partial derivative with respect to $\phi$. Here, we have used the relation

$$
\frac{\delta S_{m}}{\delta \phi}:=\sqrt{-g} \alpha T
$$

\footnotetext{
${ }^{1}$ The metric signature is $(-+++)$, while the Riemann tensor is given in terms of the Christoffel symbol by $R_{\beta \mu \nu}^{\alpha}:=\Gamma_{\beta \nu, \mu}^{\alpha}-\Gamma_{\beta \mu, \nu}^{\alpha}+\Gamma_{\sigma \mu}^{\alpha} \Gamma_{\beta \nu}^{\sigma}-\Gamma_{\sigma \nu}^{\alpha} \Gamma_{\beta \mu}^{\sigma}$.
} 
where

$$
\alpha:=\gamma_{\phi}
$$

and $T:=g^{\mu \nu} T_{\mu \nu}$ with the stress energy tensor, given by

$$
T_{\mu \nu}:=\frac{-2}{\sqrt{-g}} \frac{\delta S_{m}}{\delta g^{\mu \nu}}
$$

This form of the action is unchanged under a group of field redefinitions through a Weyl transformation. Specifically, with the new metric $g_{\mu \nu}^{\star}$ and scalar field $\varphi$, defined by

$$
\begin{aligned}
& \phi=\phi(\varphi), \\
& g_{\mu \nu}=e^{2 \Gamma(\phi(\varphi))} g_{\mu \nu}^{\star},
\end{aligned}
$$

along with the coupling function $\Gamma$ and necessary condition (1), the action (2) has the same form up to a boundary term [17, 18], i.e.,

$$
S=\int d^{4} x \frac{\sqrt{-g^{\star}}}{16 \pi G}\left(F^{\star}(\varphi) R^{\star}-\mathcal{B}^{\star}(\varphi) g^{\star \mu \nu} \partial_{\mu} \varphi \partial_{\nu} \varphi-U^{\star}(\varphi)\right)+S_{m}\left[\psi_{m}, e^{2 \gamma^{\star}(\varphi)} g_{\mu \nu}^{\star}\right],
$$

through the definition of

$$
e^{\Gamma}\left(\mathcal{B}-6 F \Gamma_{\phi}^{2}-6 F_{\phi} \Gamma_{\phi}\right)^{1 / 2} \frac{d \phi}{d \varphi}:= \pm \sqrt{\mathcal{B}^{\star}} .
$$

The equation (9) yields that

$$
\frac{d \phi}{d \varphi}= \pm \sqrt{\frac{e^{-2 \Gamma \mathcal{B}^{\star}}}{\mathcal{B}-6 F \Gamma_{\phi}^{2}-6 F_{\phi} \Gamma_{\phi}}},
$$

which should be non-zero and finite. Here, the transformed functions of $F^{\star}(\varphi), \mathcal{B}^{\star}(\varphi), U^{\star}(\varphi)$ and $\gamma^{\star}(\varphi)$ are given by

$$
\begin{aligned}
& F^{\star}(\varphi)=e^{2 \Gamma(\phi(\varphi))} F(\phi(\varphi)) \\
& \mathcal{B}^{\star}(\varphi)=e^{2 \Gamma(\phi(\varphi))} \phi_{\varphi}^{2}(\varphi)\left(\mathcal{B}(\phi(\varphi))-6 F(\phi(\varphi)) \Gamma_{\phi}^{2}(\phi(\varphi))-6 F_{\phi}(\phi(\varphi)) \Gamma_{\phi}(\phi(\varphi))\right), \\
& U^{\star}(\varphi)=e^{4 \Gamma(\phi(\varphi))} U(\phi(\varphi)) \\
& \gamma^{\star}(\varphi)=\gamma(\phi(\varphi))+\Gamma(\phi(\varphi))
\end{aligned}
$$

respectively. Two special frames of the action often used in the literature are:

The Jordan frame, which is characterized by $\gamma=0$ in (2). In this frame, matter obeys the weak equivalent principle (WEP) [6, 7, 19], meaning that freely falling objects follow 
the geodesics of the Jordan frame metric [14]. But the shortcuts of this frame violates the energy conditions. However, it is not a negative kinetic energy to be problematic, but an energy that is unbounded from below. For instance, although a negative energy is usually associated with the instability and runaway solution, the Minkowski space is stable against inhomogeneous perturbations in ST [20]. Furthermore, a positive energy theorem has been shown to hold for a special ST theory in the Jordan frame [21]. We regard the Jordan frame as the physical frame in this work.

The Einstein frame, which is characterized by $F^{\star}=1, \mathcal{B}^{\star}=$ constant and $\gamma^{\star} \neq 0$ in (8). By contrast to the Jordan one, the energy conditions are satisfied in this frame, whereas the WEP is violated [22].

\section{B. Transformation between the Jordan and Einstein Frames}

In the Jordan frame, $\mathcal{B}(\phi)=\omega(\phi) / \phi$ and $\gamma(\phi)=0$ in (2), the action is given by

$$
S=\int d^{4} x \frac{\sqrt{-g}}{16 \pi G}\left(F(\phi) R-\frac{\omega(\phi)}{\phi} g^{\mu \nu} \partial_{\mu} \phi \partial_{\nu} \phi-U(\phi)\right)+S_{m}\left[\psi_{m}, g_{\mu \nu}\right]
$$

This action can be cast into a conformal frame by a Weyl transformation

$$
g_{\mu \nu}=A^{2}(\phi) g_{\mu \nu}^{\star}
$$

where $A(\phi)=e^{\Gamma(\phi)}$ is the coupling function. We can now rewrite action (12) as

$$
\begin{aligned}
S=\int d^{4} x \frac{\sqrt{-g^{\star}}}{16 \pi G}\left(R^{\star}\right. & -6 \square^{\star}(\ln A)-6 g^{\star \mu \nu}(-1)^{2} \partial_{\mu}(\ln A) \partial_{\nu}(\ln A) \\
& \left.-\frac{\omega}{\phi} A^{2} g^{\star \mu \nu} \partial_{\mu} \phi \partial_{\nu} \phi-A^{4} U(\phi)\right)+S_{m}\left[\psi_{m}, A^{2} g_{\mu \nu}^{\star}\right],
\end{aligned}
$$

where we have used the relation of $F A^{2}=1$ to obtain the action in the Einstein frame. Consequently, by using

$$
\partial_{\mu}(\ln A)=-\frac{1}{2 F} F_{\phi} \partial_{\mu} \phi
$$

we can simplify (14) to

$$
S=\int d^{4} x \frac{\sqrt{-g^{\star}}}{16 \pi G}\left[R^{\star}-2 g^{\star \mu \nu}\left(\frac{3 F_{\phi}^{2}}{4 F^{2}}+\frac{\omega}{2 \phi F}\right) \partial_{\mu} \phi \partial_{\nu} \phi-A^{4} U(\phi)\right]+S_{m}\left[\psi_{m}, A^{2} g_{\mu \nu}^{\star}\right]
$$


up to a divergence term of $6 \square^{\star}\left(\ln A^{-1}\right)$. Through the redefinition of the scalar field with

$$
\frac{d \varphi}{d \phi}:= \pm \sqrt{\frac{3 F_{\phi}^{2}}{4 F^{2}}+\frac{\omega}{2 \phi F}},
$$

we can obtain the derivative of the canonical field $\varphi=\varphi(\phi)$ in the Einstein frame. The equation (17) is important and should satisfy the derivative constraints in (1). It will be shown that the sign can determine the range of $\varphi$ in particular models in Sec. III. As a result, (16) reads as

$$
S=\int d^{4} x \frac{\sqrt{-g^{\star}}}{16 \pi G}\left(R^{\star}-2 g^{\star \mu \nu} \partial_{\mu} \varphi \partial_{\nu} \varphi-4 V(\varphi)\right)+S_{m}\left[\psi_{m}, A^{2} g_{\mu \nu}^{\star}\right],
$$

where $V(\varphi):=A^{4} U(\phi) / 4$. Obviously, the matter fields couple non-minimally to the scalar field through $A^{2}(\phi)$ in (18).

By variating (18) with respect to $g^{\star \mu \nu}$ and $\varphi$, we derive the equations of motion of the tensor and scalar fields to be

$$
\begin{aligned}
& R_{\mu \nu}^{\star}=8 \pi G\left(T_{\mu \nu}^{\star}-\frac{1}{2} T^{\star} g_{\mu \nu}^{\star}\right)+2 \partial_{\mu} \varphi \partial_{\nu} \varphi+2 V g_{\mu \nu}^{\star}, \\
& \square^{\star} \varphi=-4 \pi G \alpha(\varphi) T^{\star}+\frac{d V}{d \varphi}
\end{aligned}
$$

respectively, where $\alpha(\varphi)$ is a function of $\varphi$ in the Einstein frame, defined by

$$
\alpha(\varphi)=\frac{d \ln A}{d \varphi}=-\frac{F_{\phi}}{2 F} \frac{d \phi}{d \varphi}
$$

which is closely related to the derivative constraints. It can be checked that the equations of motion can be read by substituting $F=1, \mathcal{B}=2, \phi=\varphi$ and $U=4 V$ as well as $\gamma=\ln A$ into (3a), (3b) and (5).

Clearly, it is non-trivial that the equations of (19) derived by variating (18) with $g^{\star \mu \nu}$ and $\varphi$ in the Einstein frame are equivalent to those from the variations of the action (12) with respect to $g^{\mu \nu}$ and $\phi$ in the Jordan frame. From the inverse function theorem, $\phi(\varphi)$ exists as long as (17) never vanishes, i.e., the derivative constraints. Hence, the solution to the scalar equation in the Einstein frame must satisfy (11). Otherwise, it is not a solution to the scalar equation in the Jordan frame, which is identified as the physical equation of motion.

There is another way to understand the derivative constraints. It is clear that $U(\phi)$ is a physical potential as it is defined in the Jordan frame, whereas $V(\varphi)$ is an auxiliary one. In 
general, if there is a potential $V(\varphi)$ in the Einstein frame, one cannot claim that a solution $\varphi$ of (19b) is physical unless $U(\phi)=4 A^{-4}(\phi) V(\varphi(\phi))$ exists and is unique. It is apparent that $V$ can be a function of $\phi$ if and only if $\varphi$ is that of $\phi$. As a result, the derivative constraints should hold.

From the derivative constraints, we can further show that

$$
\frac{d \varphi}{d \phi}>0 \text { or } \frac{d \varphi}{d \phi}<0
$$

representing the choice of the sign in the RHS of (17), which cannot cross the critical value of $d \varphi / d \phi=0$ during the evolution of the scalar field [23]. Moreover, to bear the one-to-one correspondence between $F$ and $\phi, F_{\phi}$ must be non-vanishing, so that the sign of $F_{\phi}$ cannot flip either. Under the condition of $F_{\phi} \neq 0$, the action (12) can be rewritten into that in the Brans-Dicke-Bergmann-Wagoner (BDBW) theory [24].

\section{PPN Parameters in Scalar-Tensor Theories}

As discussed in the literature [18, 23, 25 27], the deviation from GR for a ST theory in the parameterized post-Newtonian $(\mathrm{PPN})$ regime can be expressed in terms of the asymptotic value of $\alpha$ at spatial infinity as well as its successive derivatives.

At the first order of PPN, it has been shown that the coupling function is sufficient to be determined by the osculating approximation [13]

$$
\ln A=\alpha_{0}\left(\varphi-\varphi_{0}\right)+\frac{1}{2} \beta_{0}\left(\varphi-\varphi_{0}\right)^{2}
$$

and

$$
\alpha=\alpha_{0}+\beta_{0}\left(\varphi-\varphi_{0}\right)
$$

where $\varphi_{0}$ is the asymptotic value of $\varphi$ at spatial infinity and $\alpha_{0}$ and $\beta_{0}$ are constants, defined as

$$
\begin{aligned}
\alpha_{0} & :=\alpha\left(\varphi_{0}\right), \\
\beta_{0} & :=\frac{d \alpha}{d \varphi}\left(\varphi_{0}\right) .
\end{aligned}
$$

Classically, for the massive scalar field, $\varphi_{0}$ should be determined by the ground state of $\varphi$, i.e., the global minimum of the potential $V(\varphi)$, which satisfies

$$
\left.\lim _{r \rightarrow \infty} \frac{d V}{d \varphi}\right|_{\varphi=\varphi_{0}}=0
$$


and

$$
\left.\lim _{r \rightarrow \infty} \frac{d^{2} V}{d \varphi^{2}}\right|_{\varphi=\varphi_{0}}>0 .
$$

It turns out that if one expresses the potential in terms of the Taylor expansion around $\varphi=\varphi_{0}$, the coefficient of the linear term should vanish. To obtain the ground state naturally, it is convenient to consider a shifted field of

$$
\varphi^{\prime}=\varphi-\varphi_{0}
$$

which simplifies (22) to be

$$
\ln A=\alpha_{0} \varphi^{\prime}+\frac{1}{2} \beta_{0} \varphi^{\prime 2}
$$

Subsequently, we obtain

$$
\ln F=-2 \alpha_{0} \varphi^{\prime}-\beta_{0} \varphi^{\prime 2}
$$

through the help of the relation $F A^{2}=1$. Note that $\alpha(\varphi)=0$ with $\alpha_{0}=\beta_{0}=0$ leads to the result in GR with the coupling function $A$ being identically unit, implying that the scalar field couples to the Riemann curvature minimally in the Jordan frame. Consequently, the potential becomes a Taylor series of $\varphi^{\prime}$ at $\varphi^{\prime}=0$ without the linear term, given by

$$
V\left(\varphi^{\prime}\right)=V_{0}+\left.\frac{1}{2 !} \frac{d^{2} V}{d \varphi^{\prime 2}}\right|_{\varphi^{\prime}=0} \varphi^{\prime 2}+\cdots
$$

where $V_{0}:=V\left(\varphi_{0}\right)$ is the minimum of the potential $V$, which gives no contribution to the equation of motion of $\varphi^{\prime}$.

Furthermore, as a radiative coordinate [10, 29] can be constructed in the Einstein frame with the metric to be the Minkowski one asymptotically, one finds that $g_{\mu \nu}(r \rightarrow \infty) \approx A_{0}^{2} \eta_{\mu \nu}$ with $A_{0}:=A\left(\varphi_{0}\right)$ in the Jordan frame. Within the conditions (25) and (26), the ground state of the potential leads to $\varphi \rightarrow \varphi_{0}\left(\varphi^{\prime} \rightarrow 0\right)$ asymptotically, resulting in

$$
A_{0}=1
$$

As a result, (22) can be used to make sure that the Jordan frame metric $g_{\mu \nu} \approx \eta_{\mu \nu}$ is asymptotically flat. 
By following the discussions in Refs. [15, 27, 28], $\alpha_{0}\left(\beta_{0}\right)$ is positive (negative) to derive the phenomena of the spontaneous scalarization. The non-vanishing property of (1) implies that the parameter of $\alpha$ in (20) can never be zero, i.e., the $\alpha$-constraint with

$$
\alpha \neq 0
$$

which indicates that there exists an unacceptable critical value for $\varphi^{\prime}$, denoted as

$$
\varphi_{c}^{\prime}:=-\frac{\alpha_{0}}{\beta_{0}}
$$

since this value leads to $\alpha=0$ from (23). Clearly, the derivative constraints in (1) are equivalent to the $\alpha$-constraint in (32). Therefore, the forbidden value of $\varphi$ is

$$
\varphi_{c}=\varphi_{c}^{\prime}+\varphi_{0}
$$

This illustrates that the solution space of the scalar field $\varphi$ in the Einstein frame is divided into two branches by the value of $\varphi_{c}$.

\section{CONSTRAINTS ON SPECIFIC MODELS}

In a specific model, the condition (1) would manifest itself as a critical value $\varphi_{c}$. We will show that the scalar field can be nowhere equal to $\varphi_{c}$ as it cannot be a solution to the scalar equation in the Jordan frame. Hence, the solution space of the scalar field in the Einstein frame is separated by $\varphi_{c}$ into two pieces. Moreover, in the PPN regime, we demonstrate that this critical value is determined by the coefficients of the coupling function (22). In this section, we will take the general Jordan frame with the action (12) and BDBW theories as two examples for discussions. In particular, we concentrate on the potential in the Einstein frame with the form of

$$
V(\varphi)=m^{2} \varphi^{2}
$$

which has the minimum of $V_{0}=0$ at $\varphi_{0}=0$. Hereafter, we drop out the superscript of prime on $\varphi^{\prime}$ for simplicity due to $\varphi^{\prime}=\varphi$. As a result, the parabola of (29) is shown as FIG. 1. 


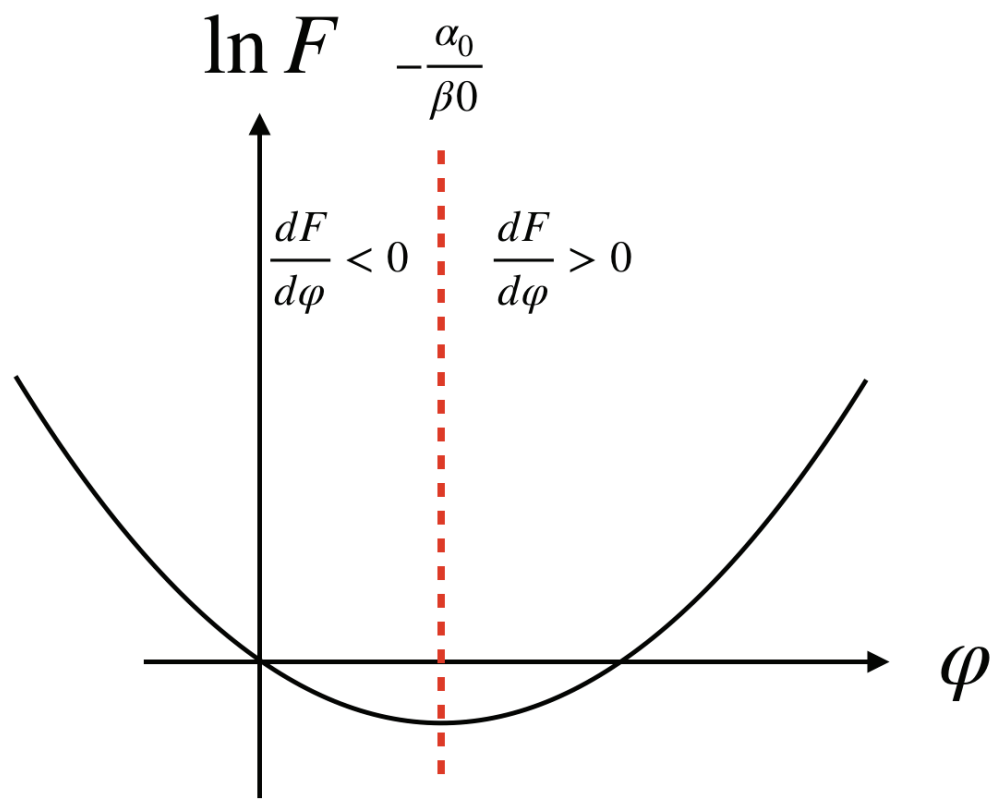

FIG. 1. The parabola reveals the relation between $\ln F$ and $\varphi$, where the dashed line represents the forbidden value $\varphi_{c}$ for $\varphi$. Particularly, we have $F=\phi$ for the BDBW theory.

\section{A. General Jordan Frame Action}

From (29) with $\varphi_{0}=0$, we get that

$$
\varphi=\varphi_{c} \pm \frac{\sqrt{\alpha_{0}^{2}-\beta_{0} \ln F}}{\beta_{0}} .
$$

With the two signs in (36), there are different relations for $\varphi(\phi)$ or $\phi(\varphi)$, resulting in different possible potentials of $U(\phi)$. Therefore, a given model in the Einstein frame may correspond to two models in the Jordan frame.

Moreover, the sign in (36) is associated with the behavior of $\varphi$. We consider two situations: (a) $\varphi$ is not always positive and (b) $\varphi$ is always positive.

For (a), like all the solutions in [15], oscillating across $\varphi=0$, we should choose the "+" sign in (36), providing that $\varphi<\varphi_{c} 2$ Therefore, once the sign in (36) is determined, the other branch of the solution space represented by $\varphi>\varphi_{c}$ should collapse, so that $\varphi_{c}$ can be viewed as a ceiling of $\varphi$. In addition, we note that the collapsed region of $\varphi>\varphi_{c}$ is independent of the potential. Any value of $\varphi$ exceeding this ceiling is not viable as it is not a solution to the scalar equation in the Jordan frame associated with the current Einstein

\footnotetext{
${ }^{2}$ If we choose the "-" sign in (36), $\varphi$ must be positive and greater than $\varphi_{c}$ due to $\beta_{0}<0$.
} 


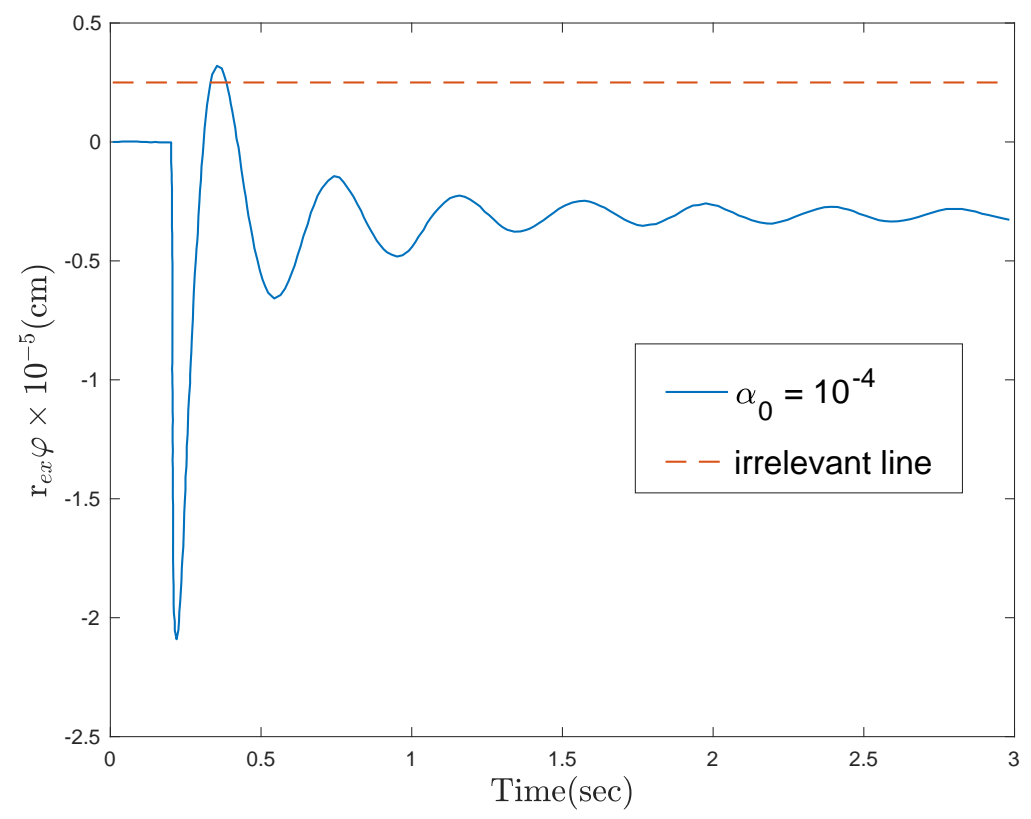

FIG. 2. Waveforms of the scalar field, extracted at $\mathrm{r}_{\mathrm{ex}}=5 \times 10^{9} \mathrm{~cm}$ away from the supernovae core in the simulation of [15], where the solid line represents that with $\alpha_{0}=10^{-4}$ in FIG. 1 of [15] and the dashed horizontal line corresponds to the critical value of $\varphi_{c}=5 \times 10^{-6}$.

frame. For example, the evolution of the scalar field in the Einstein frame during the process of the supernovae explosion has been investigated in [15]. The solution labeled by $\alpha_{0}=10^{-4}$ violates this constraint as illustrated in FIG. 2,

Although the solution space is constrained by $\varphi<\varphi_{c}$, we still cannot specify a unique model in the Jordan frame due to the lack of the information about $F_{\phi}$ to determine the form of the relation $\phi(\varphi)$. However, we can constrain $\alpha$ to be positive as $\varphi<\varphi_{c}$, which gives rise to

$$
F_{\phi} \frac{d \phi}{d \varphi}<0
$$

due to (20). Clearly, the positivity of $F_{\phi}$ implies that the sign of (17) should be minus and vice versa. Furthermore, we have two models in the Jordan frame based on the " \pm " signs of $F_{\phi}$, within $\varphi<\varphi_{c}$. For (b), there are two cases. The first one corresponds to the "-" sign in (36) with $\varphi>\varphi_{c}$. Similar to the argument above, there exists two models in the Jordan frame related to the sign of $F_{\phi}$. In this case with the equivalence of $\alpha<0$, we have 
the relation

$$
F_{\phi} \frac{d \phi}{d \varphi}>0
$$

By contrast, the case for the "+" sign requires $0<\varphi<\varphi_{c}$. The solution space is bounded, which is the most restrictive one among the cases.

By differentiating both sides of (36) with respect to $\phi$, one gets an additional equation

$$
\frac{d \varphi}{d \phi}=\frac{\mp F_{\phi}}{2 F \sqrt{\alpha_{0}^{2}-\beta_{0} \ln F}}
$$

which suggests that $F_{\phi}$ determines the sign of $d \varphi / d \phi$ since $F>0$. We note that the sign in (39) is opposite to the one chosen in (36). The requirement of $\ln F>\alpha_{0}^{2} / \beta_{0}$ to prevent $\varphi$ from being imaginary is fulfilled because (29) has minimum $\alpha_{0}^{2} / \beta_{0}$ at the forbidden point $\varphi_{c}$, which can easily be checked from FIG. 1. One can see that the discussions of the sign problem of $F_{\phi}$ above are consistent with the sign in (39) .

\section{B. Brans-Dicke-Bergmann-Wagoner theory}

Another example is the BDBW theory with $F=\phi$ in the action (12), particularly given by

$$
S=\int d^{4} x \frac{\sqrt{-g}}{16 \pi G}\left(\phi R-\frac{\omega(\phi)}{\phi} g^{\mu \nu} \partial_{\mu} \phi \partial_{\nu} \phi-U(\phi)\right)+S_{m}\left[\psi_{m}, g^{\mu \nu}\right] .
$$

It can be found that the sign of $d \varphi / d \phi$ can totally be determined through $\alpha$ due to $F_{\phi}=1$. In this subsection, we will transform the potential defined in the Einstein frame back into the Jordan one and discuss the ambiguity arising from this transformation. In this specific case, since $F(\phi)=A^{-2}$ is simply $\phi$, we have

$$
\phi=A^{-2}>0
$$

leading to

$$
\ln \phi=-2 \alpha_{0} \varphi-\beta_{0} \varphi^{2}
$$

which is also illustrated in FIG. 1.

From FIG. 1, it is easy to see that the condition (1) is equivalent to $\varphi \neq \varphi_{c}$. As a result, one finds that (23) can never be zero. Consequently, (20) reads as

$$
\alpha=-\frac{1}{2 \phi} \frac{d \phi}{d \varphi} \text {. }
$$


From (43), we obtain the inequality by (41)

$$
\alpha \frac{d \varphi}{d \phi}=-\frac{1}{2 \phi}<0
$$

As mentioned early that $d \varphi / d \phi$ is either positive or negative, the valid value of $\varphi$ can fix the sign in (17) as follows. For $\varphi>\varphi_{c}$, we have $\alpha<0$, so that one has

$$
\frac{d \varphi}{d \phi}=\frac{\sqrt{3+2 \omega}}{2 \phi}
$$

by (44). Similarly, the redefinition of the scalar fields leads to

$$
\frac{d \varphi}{d \phi}=-\frac{\sqrt{3+2 \omega}}{2 \phi}
$$

for $\varphi<\varphi_{c}$.

In the limit of the BD theory, $\omega$ in (40) is constant, i.e., $\omega(\phi)=\omega_{\mathrm{BD}}$. Consequently, the equation in (43) reveals that

$$
\alpha=\alpha_{0}+\beta_{0} \varphi=\mp \frac{1}{\sqrt{3+2 \omega_{\mathrm{BD}}}}
$$

where the minus (plus) case corresponds to the branch of $\varphi>\varphi_{c}\left(\varphi<\varphi_{c}\right)$. Since the right-hand side of (47) is constant, we have two possibilities: (i) $\beta_{0}=0$ and (ii) $\varphi$ is almost a constant. Due to the negativity of $\beta_{0}$ in this paper, the solution is restricted to the second one.

It is interesting to note that $\omega_{\mathrm{BD}} \rightarrow \infty$ is a critical value, equivalent to $\varphi=\varphi_{c}$ due to $\alpha=0$. Even though this case is consistent with those in the literature and in turn reproduces the results in GR in the sense that all its predictions become indistinguishable from GR in the Jordan frame [30], it cannot be transformed to the Einstein frame due to the violation of the derivative constraints and $\alpha$-constraint. Therefore, the case of the infinite $\omega_{\mathrm{BD}}$ is improper to be discussed in the Einstein frame, which shows the inequivalence between the two frames.

We again note that the critical value of $\varphi_{c}$ is so generic that the results of all the simulations should obey the criterion $\varphi \neq \varphi_{c}$. For instance, it has been concentrated on the BDBW theory to investigate the dynamical scalarization of the neutron star binaries in ST in [31]. The critical value for the scalar field in their setting is $\varphi_{c}=0$, and the results therein do all satisfy this criterion, i.e., no crossing the line of $\varphi=0$. 
Starting from (42) in the BDBW theory, we get

$$
\varphi=\varphi_{c} \pm \frac{\sqrt{\alpha_{0}^{2}-\beta_{0} \ln \phi}}{\beta_{0}} .
$$

Since $U=4 V A^{-4}=4 m^{2} \varphi^{2} \phi^{2}$ as defined in (18), we recover the potential $U$ in the Jordan frame, given by

$$
U(\phi)=4 m^{2} \beta_{0}^{-2}\left(2 \alpha_{0}^{2}-\beta_{0} \ln \phi \mp 2 \alpha_{0} \sqrt{\alpha_{0}^{2}-\beta_{0} \ln \phi}\right) \phi^{2}
$$

and

$$
U(\varphi)=4 m^{2} \varphi^{2} \exp \left(-4 \alpha_{0} \varphi-2 \beta_{0} \varphi^{2}\right)
$$

in terms of $\phi$ and $\varphi$, respectively, indicating two different potentials in the Jordan frame, which depend on the branches of the scalar field in the Einstein frame, i.e., the sign chosen in (48). For the case of the action (12) in Sec. IIIA, one cannot consider only the "+" sign in (17), which is equivalent to choose one model from two prospective ones.

In addition, the undetermined signs in (48) can be eliminated by considering the function of $\left(\varphi-\varphi_{c}\right)^{2}$. Hence, the solution space has an $\mathbb{Z}_{2}$ symmetry with respect to $\varphi_{c}$. In other words, the model in the Jordan frame, which is responsible for the given model in the Einstein frame, remains the same under the transformation $\varphi-\varphi_{c} \longleftrightarrow-\left(\varphi-\varphi_{c}\right)$. We can shift $\varphi$ to $\bar{\varphi}=\varphi-\varphi_{c}$, resulting in that the existence of the $\mathbb{Z}_{2}$ symmetry is characterized by an even potential $U(\bar{\varphi})$ of $\bar{\varphi}$. The potential (50), which can be rewritten as

$$
U(\bar{\varphi})=4 m^{2}\left(\bar{\varphi}+\varphi_{c}\right)^{2} \exp \left[-2 \beta_{0}\left(\bar{\varphi}^{2}-\varphi_{c}^{2}\right)\right]
$$

is not a even function of $\bar{\varphi}$ apparently. As a result, the one to one correspondence between models in both frames holds strictly. In general, it is hard to have the same symmetry of $\mathbb{Z}_{2}$ at $\varphi_{c}$ for $U(\varphi)$. Particularly, the direct effect of the $\mathbb{Z}_{2}$ symmetry at $\varphi_{c}$ for the potential is that the model in the Einstein frame is associated with a unique model in the Jordan frame.

\section{CONCLUSIONS}

In order to use the formulation of the ST theories in the Einstein frame, we have investigated the redefinition of the scalar. We have found that the regularity of such redefinition (17), which comes from the requirement $d \varphi / d \phi \neq 0$ and has been shown to be equivalent 
to the condition $\alpha \neq 0$, gives an irrelevant value $\varphi_{c}$ of the scalar field in the Einstein frame. The value of $\varphi_{c}$ separates the solution space of the scalar field into two viable regions. Any scalar field in the Einstein frame, which crosses the irrelevant value, cannot be the solution in the Jordan frame.

The two signs in (17) result in two different models in the Jordan frame. In some special cases, such as that in the BDBW theory without a potential, these two models in the Jordan frame coincide with each other. In general, the sign of (17) can be obtained by $F_{\phi}$ and $\varphi$. For the case with one branch of the solution space collapsed, the sign of (17) can be fixed by the sign of $F_{\phi}$ alone. In such a case, it is the ambiguity of the sign of $F_{\phi}$ that gives rise to two prospective models in the Jordan frame. On the other hand, in some models with $F_{\phi}$ being constant, such as the BDBW theory, the sign of (17) is fully determined by the branch of the scalar fields. If we consider the asymptotic value $\varphi_{0} \neq 0$, (36) should be modified as

$$
\varphi-\varphi_{0}=\varphi_{c}^{\prime} \pm \frac{\sqrt{\alpha_{0}^{2}-\beta_{0} \ln F}}{\beta_{0}} .
$$

Together with (34), one can also conclude that if the potential $U(\varphi)$ in the Jordan frame is an even function with respect to the value of $\varphi=\varphi_{c}$, the correspondence between the models in two frames is one to one. Hence, by satisfying the $\alpha$-constraint in (32) the results in the Einstein frame can be well-defined under this condition.

We have demonstrated that the critical value $\varphi_{c}$ provides a viable constraint on the formulation of the ST theories in the Einstein frame, which is even independent of the form of the potential. Clearly, all the numerical results must obey the criterion of no crossing the line of the critical value. Otherwise, they will not have any physical meaning in the Einstein frame. In addition, we have also shown that the case of the infinite $\omega_{\mathrm{BD}}$ in the BD theory in the Einstein frame is irrelevant due to the violation of the derivative constraints and $\alpha$-constraint.

Furthermore, for the case without a potential, two branches of the solution space of the scalar field in the Einstein frame correspond to the same model in the Jordan one. However, the solution branch of $\varphi$ should be specified because the $\alpha$-constraint is strict with the behavior of $\varphi$ during the evolution in the Einstein frame.

Finally, we conclude that the critical value $\varphi_{c}$ induced from the $\alpha$-constraint or derivative constraints and the sign determination in (17) are two important issues to study the ST theory in the Einstein frame. 


\section{ACKNOWLEDGMENTS}

We would like to thank Patrick Chi-Kit Cheong for useful discussions. The work was partially supported by National Center for Theoretical Sciences, Ministry of Science and Technology (MoST-107-2119-M-007-013-MY3 and MoST-108-2811-M-001-598), and Academia Sinica Career Development Award Program (AS-CDA-105-M06).

[1] P. Jordan, Schwerkraft und weltall, Vieweg (1955).

[2] P. Jordan, Z. Phys. 157, 112 (1959).

[3] P. G. Bergmann, Annals of Mathematics, 255-264 (1948).

[4] M. Fierz, Helv. Phys. Acta 29, 128 (1956).

[5] T. Kaluza, Sitzungsber. Preuss. Akad. Wiss. Berlin (Math. Phys. ) 1921, 966 (1921); O. Klein, Z. Phys. 37, 895 (1926) [Surveys High Energ. Phys. 5, 241 (1986)].

[6] C. Brans and R. H. Dicke, Phys. Rev. 124, 925 (1961).

[7] R. H. Dicke, Phys. Rev. 125, 2163 (1962).

[8] P. G. Bergmann, Int. J. Theor. Phys. 1, 25 (1968).

[9] R. V. Wagoner, Phys. Rev. D 1, 3209 (1970).

[10] T. Damour and G. Esposito-Farese, Class. Quant. Grav. 9, 2093 (1992).

[11] V. Faraoni and S. Nadeau, Phys. Rev. D 75, 023501 (2007)

[12] S. Capozziello, P. Martin-Moruno and C. Rubano, Phys. Lett. B 689, 117 (2010).

[13] T. Damour, arXiv:0704.0749 [gr-qc].

[14] Y. Fujii and K. Maeda, The Scalar-Tensor Theory of Gravitation (Cambridge University Press, Cambridge, England, 2007).

[15] U. Sperhake, C. J. Moore, R. Rosca, M. Agathos, D. Gerosa and C. D. Ott, Phys. Rev. Lett. 119, no. 20, 201103 (2017).

[16] C. Q. Geng, H. J. Kuan, L. W. Luo, in preparation.

[17] E. E. Flanagan, Class. Quant. Grav. 21, 3817 (2004).

[18] L. Jrv, P. Kuusk, M. Saal and O. Vilson, J. Phys. Conf. Ser. 532, 012011 (2014).

[19] M. Salgado, Class. Quant. Grav. 23, 4719 (2006).

[20] V. Faraoni, Phys. Rev. D 70, 081501 (2004). 
[21] O. Bertolami, Phys. Lett. B 186, 161 (1987).

[22] C. H. Brans, gr-qc/0506063 (2005).

[23] L. Jarv, P. Kuusk and M. Saal, Phys. Rev. D 76, 103506 (2007).

[24] T. Clifton, P. G. Ferreira, A. Padilla and C. Skordis, Phys. Rept. 513, 1 (2012).

[25] T. Damour and G. Esposito-Farese, Phys. Rev. D 53, 5541 (1996).

[26] T. Damour and G. Esposito-Farese, Phys. Rev. D 54, 1474 (1996).

[27] T. Damour and G. Esposito-Farese, Phys. Rev. Lett. 70, 2220 (1993).

[28] J. Novak, Phys. Rev. D 58, 064019 (1998).

[29] L. Blanchet, Proc. Roy. Soc. Lond. A 409, 383 (1987).

[30] C. M. Will, Living Rev. Rel. 17, 4 (2014).

[31] C. Palenzuela, E. Barausse, M. Ponce and L. Lehner, Phys. Rev. D 89, no. 4, 044024 (2014). 